\title{
Analysis of Instantaneous Center of Zero Acceleration of Rigid Body in Planar Motion
}

\author{
Haibin Sun (Corresponding author) \& Tingting Liu \\ Department of Physics and Electronics \\ Taishan University \\ Shandong 271021, China \\ E-mail: sunhbphy@hotmail.com
}

\begin{abstract}
When the angular velocity vector of rigid body in the plane motion unequal to zero, we can certainly find a point which instantaneous acceleration equal to zero at that particular instant. The point is named instantaneous center of zero acceleration. We proved the existence of the point and obtained the location of the point. We could solve mechanical problem quickly using instantaneous center of acceleration as base point.
\end{abstract}

Keywords: Instantaneous center of zero acceleration, Rigid body, Planar motion

\section{Introduction}

Let us analyze a rigid body which is in planar motion. It is clear that if we could find a point, $C$, in the rigid body for which the instantaneous velocity is zero, then the velocity of the body at that particular instant would consist only of a pure rotation of the body about that point (no translation)(Fredy R. Zypman,2005). If we choose the point $C$ which is momentarily stationary at the instant considered as a base point, the velocity equation for an arbitrary point $A$ in the body simplifies to

$$
\boldsymbol{v}_{\mathrm{A}}=\boldsymbol{\omega} \times \boldsymbol{r}_{\mathrm{A}},
$$

where $\omega$ is the body angular velocity (J. F. LI,2001). If we know the angular velocity, and the radius vector of point $A$ with respect to $C$, then we could determine the velocity of point $A$. Point $C$ can be taken as the center of rotation only at the given instant, and is therefore known as the instantaneous center of zero velocity. If we can determine the instantaneous center of zero acceleration, we can solve some mechanical problems efficiently.

\section{Deduction of instantaneous center of zero acceleration}

The acceleration of an arbitrary point $P$ in the rigid body is (SH M CH, 2001)

$$
\boldsymbol{a}=\boldsymbol{a}_{\mathrm{A}}+\frac{d \boldsymbol{\omega}}{d t} \times\left(\boldsymbol{r}-\boldsymbol{r}_{0}\right)-\omega^{2}\left(\boldsymbol{r}-\boldsymbol{r}_{0}\right) .
$$

Here, $A$ is a base point, $\boldsymbol{r}$ is the radius vector of the point considered with respect to the fixed point $O, \boldsymbol{r}_{0}$ is the radius vector of the point $A$ with respect to the point $O, r$ ' is the radius vector of the point with respect to the point $A$, and $\boldsymbol{r}^{\prime}=\boldsymbol{r}-\boldsymbol{r}_{0} ; \boldsymbol{\omega}$ is the angular velocity, $\alpha=\frac{d \omega}{d t}$ is the angular acceleration. The directions of the angular velocity and angular acceleration indicate the axis of rotation. Consider the base point $A$ and the point $P$ having coordinates $\left(\xi_{\mathrm{o}}, \eta_{\mathrm{o}}\right)$ and $(\xi, \eta)$ in a fixed Cartesian coordinate system, $o-\xi \eta$. In Cartesian coordinate system, $A-x y$, which is a body fixed reference system, the point $P$ having coordinates $(x, y)$. Take $Z$ axis to be the fixed direction perpendicular to the planar motion. The position of point $A$ and $P$ are illustrated in Fig. 1.

\subsection{Existence proof}

When the rigid body moves in plane with angular velocity, $\omega$, which is unequal to zero, we choose a point, $C$, in the body for which the instantaneous acceleration is zero. We define this point as the instantaneous center of zero acceleration. From Eq. (2), we have

$$
0=\boldsymbol{a}_{\mathrm{A}}+\frac{d \omega}{d t} \times\left(\boldsymbol{r}_{\mathrm{c}}-\boldsymbol{r}_{0}\right)-\omega^{2}\left(\boldsymbol{r}_{\mathrm{c}}-\boldsymbol{r}_{0}\right),
$$


where, $\boldsymbol{r}_{\mathrm{c}}=\xi_{c} \boldsymbol{i}+\eta_{c} \boldsymbol{j}$ is the radius vector of the instantaneous center of zero acceleration with respect to the origin $O$.

Taking the scalar product of Eq. (2) with $\omega$, we obtain

$$
0=\omega \cdot \boldsymbol{a}_{\mathrm{A}}+\omega \cdot\left[\frac{d \omega}{d t} \times\left(\boldsymbol{r}_{\mathrm{c}}-\boldsymbol{r}_{0}\right)\right]-\omega \cdot\left[\omega^{2}\left(\boldsymbol{r}_{\mathrm{c}}-\boldsymbol{r}_{0}\right)\right] .
$$

The direction of angular velocity vector, $\omega$, is parallel to the direction of angular acceleration vector, $\alpha=\frac{d \omega}{d t}$. So $\omega$ is orthogonal to $\frac{d \omega}{d t} \times\left(\boldsymbol{r}_{\mathrm{c}}-\boldsymbol{r}_{0}\right)$. Hence,

$$
\omega \cdot\left[\frac{d \omega}{d t} \times\left(\boldsymbol{r}_{\mathrm{c}}-\boldsymbol{r}_{0}\right)\right]=0 .
$$

In fact, the angular velocity, $\omega$, is perpendicular to the plane that the body is moving, and the relative radius vector, $\boldsymbol{r}_{\mathrm{c}}-\boldsymbol{r}_{0}$, also lies in this plane. So the vector angular velocity, $\boldsymbol{\omega}$, is perpendicular to the vector $\boldsymbol{r}_{\mathrm{c}}-\boldsymbol{r}_{0}$. Therefore, we have

$$
\omega \cdot\left[\omega^{2}\left(\boldsymbol{r}_{\mathrm{c}}-\boldsymbol{r}_{0}\right)\right]=0
$$

Substituting Eqs. (5) and (6) into Eq. (4), we obtain

$$
\omega \cdot a_{\mathrm{A}}=0 .
$$

In the case, the acceleration of any point in the rigid body, $\boldsymbol{a}_{\mathrm{A}}$, must be orthogonal to the angular velocity, $\boldsymbol{\omega}$. Surely Eq. (7) is an identity.

In conclusion, when the rigid body moves in plane with angular velocity, $\omega$, that is unequal to zero, we can certainly find the point , $C$, in the body for which the instantaneous acceleration is zero, i.e., the instantaneous center of zero acceleration must exist.

2.2 Position of the instantaneous center of zero acceleration

Taking the vector product of Eq. (2) with $\omega$, we obtain

$$
0=\boldsymbol{\omega} \times \boldsymbol{a}_{\mathrm{A}}+\boldsymbol{\omega} \times\left[\frac{d \boldsymbol{\omega}}{d t} \times\left(\boldsymbol{r}_{\mathrm{c}}-\boldsymbol{r}_{0}\right)\right]-\boldsymbol{\omega} \times\left[\omega^{2}\left(\boldsymbol{r}_{\mathrm{c}}-\boldsymbol{r}_{0}\right)\right] .
$$

Additionally,

$$
\omega \times\left[\frac{d \omega}{d t} \times\left(r_{\mathrm{c}}-\boldsymbol{r}_{0}\right)\right]=\alpha\left[\left(\boldsymbol{r}_{\mathrm{c}}-\boldsymbol{r}_{0}\right) \cdot \boldsymbol{\omega}\right]-\left(\boldsymbol{r}_{\mathrm{c}}-\boldsymbol{r}_{0}\right)(\boldsymbol{\omega} \cdot \boldsymbol{\alpha})=-\left(\boldsymbol{r}_{\mathrm{c}}-\boldsymbol{r}_{0}\right)(\boldsymbol{\omega} \cdot \boldsymbol{\alpha}) .
$$

Here, we have used the vector identity: $\boldsymbol{A} \times(\boldsymbol{B} \times \boldsymbol{C})=(\boldsymbol{A} \cdot \boldsymbol{C}) \boldsymbol{B}-(\boldsymbol{A} \cdot \boldsymbol{B}) \boldsymbol{C}(\mathrm{K} . \mathrm{F}$. Riley, 2002).

Substituting Eq. (9) into Eq. (8), we have

$$
0=\boldsymbol{\omega} \times \boldsymbol{a}_{\mathrm{A}}-\left(\boldsymbol{r}_{\mathrm{c}}-\boldsymbol{r}_{0}\right)(\boldsymbol{\omega} \cdot \boldsymbol{\alpha})-\left(\boldsymbol{r}_{\mathrm{c}}-\boldsymbol{r}_{0}\right)(\boldsymbol{\omega} \cdot \boldsymbol{\alpha}) .
$$

From Eq. (10), we can obtain the radius vector of the instantaneous center of zero acceleration. The calculation of $\boldsymbol{r}_{\mathrm{c}}$, in this case, requires knowing $\boldsymbol{r}_{0}, \boldsymbol{a}_{\mathrm{A}}, \boldsymbol{\omega}$ and $\boldsymbol{\alpha}$. In general, the angular acceleration vector, $\boldsymbol{\alpha}$, is unknown to us. Therefore, the location of the instantaneous center of zero acceleration is difficult to locate.

In the fixed Cartesian coordinate system $o-\xi \eta$, Eq. (10) can be written as

$$
\left\{\begin{array}{l}
\xi_{c}=\xi_{0}+\frac{\omega^{2} a_{A \xi}-\alpha a_{A \eta}}{\alpha^{2}+\omega^{4}} \\
\eta_{c}=\eta_{0}+\frac{\alpha a_{A \xi}+\omega^{2} a_{A \eta}}{\alpha^{2}+\omega^{4}}
\end{array},\right.
$$

In the moving Cartesian coordinate system $A-x y$, Eq. (10) can be written as 


$$
\left\{\begin{array}{c}
x_{c}=\frac{\omega^{2} a_{A x}-\alpha a_{A y}}{\alpha^{2}+\omega^{4}} \\
y_{c}=\frac{\alpha a_{A x}+\omega^{2} a_{A y}}{\alpha^{2}+\omega^{4}}
\end{array}\right.
$$

It should be noted that the location of the instantaneous center of zero acceleration will change in time. The path described by the instantaneous center is called the space centrode, and the locus of the positions of the instantaneous centers on the body is called the body centrode.

\section{Application of the instantaneous center of zero acceleration}

\subsection{Acceleration of any point in the rigid body}

If we define the instantaneous center of zero acceleration, $C$, as the base point, the acceleration of any point, $D$, in the body can be obtained from Eq. (2),

$$
\boldsymbol{a}_{D}=\frac{d \omega}{d t} \times\left(\boldsymbol{r}-\boldsymbol{r}_{c}\right)-\omega^{2}\left(\boldsymbol{r}-\boldsymbol{r}_{c}\right)=\frac{d\left[\boldsymbol{\omega} \times\left(\boldsymbol{r}-\boldsymbol{r}_{c}\right)\right]}{d t} .
$$

Here, $\frac{d \omega}{d t} \times\left(\boldsymbol{r}-\boldsymbol{r}_{\mathrm{c}}\right)$, is the tangential component of the acceleration with respect to the instantaneous center, and, $-\omega^{2}\left(\boldsymbol{r}-\boldsymbol{r}_{c}\right)$, is the normal component of the acceleration with respect to the instantaneous center. The above equation is analogous to the equation expressing the acceleration to fixed axis rotation. So we can say that the acceleration of the body at that particular instant would consist only of a pure rotation of the body about the instantaneous center of acceleration.

We choose the instantaneous center of acceleration as the origin of the Cartesian coordinate, in this coordinate system, Eq. (13) has the form

$$
\boldsymbol{a}_{D}=\frac{d \boldsymbol{\omega}}{d t} \times \boldsymbol{r}-\omega^{2} \boldsymbol{r}=\frac{d(\boldsymbol{\omega} \times \boldsymbol{r})}{d t},
$$

where $\boldsymbol{r}=\overrightarrow{C D}=x \boldsymbol{i}+y \boldsymbol{j}$ is the radius vector of point $D$ with respect to the instantaneous center, $C$ (cf. Fig. 2).

Assume that the angular velocity, $\omega$, and the angular acceleration, $\alpha=\frac{d \omega}{d t}$, have the same direction. In component form,

Eq. (14) can be written as

$$
\left\{\begin{array}{l}
a_{D x}=-\omega^{2} x-\alpha y \\
a_{D y}=\alpha x-\omega^{2} y
\end{array},\right.
$$

\subsection{Location of the instantaneous center of acceleration}

At a particular instant, the angular velocity, $\omega$, the angular acceleration, $\alpha=\frac{d \omega}{d t}$, and the acceleration of point $D, \boldsymbol{a}_{D}=$ $\boldsymbol{a}_{D x} \boldsymbol{i}+\boldsymbol{a}_{\mathrm{D} y} \boldsymbol{j}$, all have certain values. Solving Eqs. (15), we have

$$
\left\{\begin{array}{c}
x=-\frac{\omega^{2} a_{D x}-\alpha a_{D y}}{\alpha^{2}+\omega^{4}} \\
y=-\frac{\alpha a_{D x}+\omega^{2} a_{D y}}{\alpha^{2}+\omega^{4}}
\end{array}\right.
$$

From Eqs. (16), we can obtain the distance between point $D$ and instant center $C$ :

$$
|\boldsymbol{r}|=\overline{C D}=\sqrt{x^{2}+y^{2}}=\frac{a_{D}}{\sqrt{\alpha^{2}+\omega^{4}}} .
$$

Here, $a_{D}=\sqrt{a_{D x}^{2}+a_{D y}^{2}}$, is the magnitude of acceleration $\boldsymbol{a}_{D}$. 
The angle, $\varphi$, between the direction of acceleration vector $\boldsymbol{a}_{D}$ and the direction of radius vector $\boldsymbol{r}$ is

$$
\varphi=\arctan \frac{\alpha}{\omega^{2}} .
$$

Here, if $\alpha>0, \varphi$ is a positive acute angle, i.e., counterclockwise to point $D$; otherwise, $\varphi$ clockwise to point $D$.

According to Eqs. (17) and (18), if we know the values of $\omega, \alpha$ and $a_{D}$, we can calculate the angle, $\varphi$, and the magnitude of $\overrightarrow{C D}$. Then we can draw the location of the instantaneous center of zero acceleration, $C$ (Ch $\mathrm{H} \mathrm{Ch}, 1999)$. See Fig. 3.

3.3 Theorem of relative angular momentum about the instantaneous center

The equation of relative angular momentum of a rigid body which is in planar motion is (H. E. Williams, 2000)

$$
\boldsymbol{M}_{A}=\frac{\boldsymbol{d} \boldsymbol{L}_{A}}{\boldsymbol{d} t}+m \boldsymbol{r}_{C A} \times \boldsymbol{a}_{A} .
$$

Here, $\boldsymbol{M}_{\boldsymbol{A}}$ is the net moment about $A, \boldsymbol{L}_{\boldsymbol{A}}$ is the relative angular momentum about $A, \boldsymbol{r}_{C A}$ is the radius vector of point $C$ about $\mathrm{A}$ and $\boldsymbol{a}_{\boldsymbol{A}}$ is the acceleration of point $A$.

When we define the instantaneous center of zero acceleration, $C$, as the base point, the theorem of relative angular momentum with respect to the instantaneous center can be expressed as

$$
\boldsymbol{M}_{\mathrm{c}}=\frac{d \boldsymbol{L}}{d t}=I \alpha
$$

Here, $\boldsymbol{M}_{\mathrm{c}}$ is the net moment about point $C$ of all external forces acting on the body, $\boldsymbol{L}$ is the relative angular momentum about $C$ of the body, $I$ is the moment of inertia about $C, \alpha$ is the angular acceleration of the body.

\section{Conclusion}

On the basis of vector algebra, we have proved the existence of the instantaneous center of zero acceleration, and calculated the location of the instantaneous center of acceleration. The instantaneous center of zero acceleration plays an important role in solving mechanical problems.

\section{References}

Ch H Ch. (1999). Analyses on Instantaneous Acceleration Center of Rigid Bodies Planner Motion.Journal of Ji'mei University(natural Science), Vol. 4,No. 1:19-22.

Fredy R. Zypman. (2005). Instantaneous center of rotation and centrodes: background and new examples. Int. J. Mech. Enging. Educ., 35 (1):79-90.

H. E. Williams. (2000). A note on the use of the instant center as a reference point for angular momentum theorems. Int. J. Mech. Enging. Educ., 28 (2):185-186.

J. F. LI, X. Zhang. (2001). Classical Mechanics. Tsinghua University Press, Beijing, 30.

K. F. Riley, M. P. Hobson and S. J. Bence. (2002). Mathematical Methods for Physics and Engineering (second edition). Cambridge University Press, Cambridge, 230.

SH M CH. (2001). Theoretical Mechanics. Higher Education Press, Beijing, 117. 


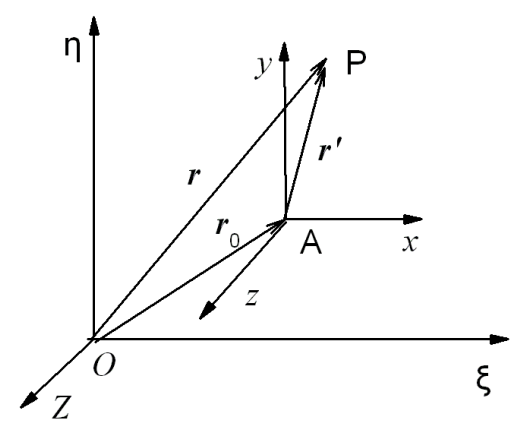

Fig. 1. The position of any point $P$ in a rigid body which is in planar motion.

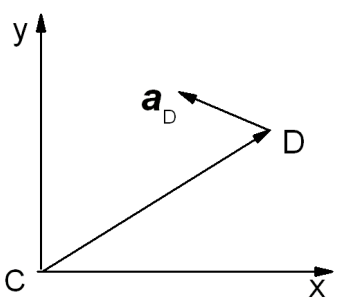

Fig. 2. Acceleration of any point $D$ with respect to the instantaneous center of zero acceleration C.

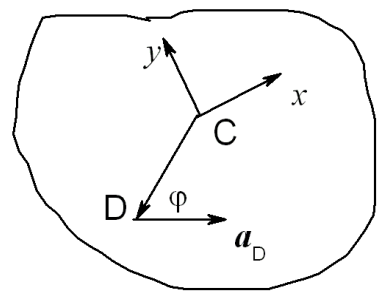

Fig. 3. Location of the instantaneous center of zero acceleration given the direction and magnitude of acceleration of any point. 